\title{
Principal Typings for Explicit Substitutions Calculi $^{\star}$
}

\author{
Daniel Lima Ventura ${ }^{1 \star \star}$, Mauricio Ayala-Rincón ${ }^{1 \star \star \star}$, and \\ Fairouz Kamareddine ${ }^{2}$ \\ ${ }^{1}$ Grupo de Teoria da Computação, Dep. de Matemática Universidade de Brasília, \\ Brasília D.F., Brasil \\ 2 School of Mathematical and Computer Sciences Heriot-Watt University, \\ Edinburgh, Scotland UK \\ \{ventura, ayala\}@mat.unb.br, fairouz@macs.hw.ac.uk
}

\begin{abstract}
Having principal typings (for short PT) is an important property of type systems. In simply typed systems, this property guarantees the possibility of a complete and terminating type inference mechanism. It is well-known that the simply typed $\lambda$-calculus has this property but recently J.B. Wells has introduced a system-independent definition of PT, which allows to prove that some type systems, e.g. the Hindley/Milner type system, do not satisfy PT. Explicit substitutions address a major computational drawback of the $\lambda$-calculus and allow the explicit treatment of the substitution operation to formally correspond to its implementation. Several extensions of the $\lambda$-calculus with explicit substitution have been given but some of which do not preserve basic properties such as the preservation of strong normalization. We consider two systems of explicit substitutions $\left(\lambda s_{e}\right.$ and $\left.\lambda \sigma\right)$ and show that they can be accommodated with an adequate notion of PT. Specifically, our results are as follows:

- We introduce PT notions for the simply typed versions of the $\lambda s_{e^{-}}$and the $\lambda \sigma$-calculi and prove that they agree with Wells' notion of PT.

- We show that these versions satisfy PT by revisiting previously introduced type inference algorithms.
\end{abstract}

Key Words: lambda-calculus, explicit substitution, principal typings

\section{Introduction}

The development of well-behaved calculi of explicit substitutions is of great interest in order to bridge the formal study of the $\lambda$-calculus and its real implementations. Since $\beta$ contraction depends on the definition of the operation

^ Research supported by the CNPq Brazilian Research Council.

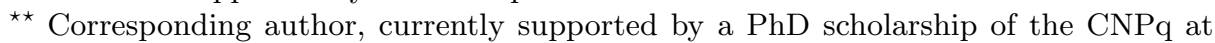
the Heriot-Watt University.

$\star \star \star$ Author partially supported by the CNPq. 
of substitution, which is informally given in the theory of $\lambda$-calculus, substitutions are in fact made explicit, but obscurely developed (that is, in an empirical manner), when most computational environments based on the $\lambda$-calculus are implemented. A remarkable exception is $\lambda$ Prolog, for which its explicit substitutions calculus, the suspension calculus, has been extracted and formally studied [NaWi98].

In the study of making substitutions explicit, several alternatives rose out and all of them are directed to guarantee essential properties such as simulating beta-reduction, confluence, noetherianity (of the associated substitution calculus), subject reduction, having principal typings (for short PT), preservation of strong normalization etc. This is a non trivial task; for instance, the $\lambda \sigma$-calculus [ACCL91], that is one of the first proposed calculi of explicit substitutions, was reported to break the latter property some years after its introduction [Mel95]: this implies that infinite derivations starting from well-typed $\lambda$-terms are possible in this calculus, which is at least questionable for any mechanism supposed to simulate the $\lambda$-calculus explicitly. In this paper, the focus is on the PT property, which means that for any typable term $M$, there exists a type judgment $A \vdash M: \tau$, representing all possible typings $\left\langle A^{\prime}, \tau^{\prime}\right\rangle$ for $M$. In the simply typed $\lambda$-calculus this corresponds to the existence of more representative typings. PT guarantees compositional type inference helping to find a complete/terminating type inference algorithm.

In section 2 we present the type-free $\lambda$-calculus in de Bruijn notation, the $\lambda s_{e}$-calculus [KR97] and the $\lambda \sigma$-calculus [ACCL91]. In section 3 we present the type assignment systems background and then we present simply typed systems for each calculus we study. Then, we discuss the general notion of principal typings defined in [We2002] and present notions of principal typings for the $\lambda$ calculus in de Bruijn notation, the $\lambda \sigma$ - and the $\lambda s_{e}$-calculi and prove that they are adequate. In section 4 we conclude and present future work.

\section{The type free calculi}

\section{$2.1 \lambda$-calculus in de Bruijn notation}

Definition 1 (Set $\left.\Lambda_{d B}\right)$. The syntax of $\lambda$-calculus in de Bruijn notation, the $\lambda d B$-calculus, is defined inductively as

Terms $M::=\underline{n}|(M M)| \lambda . M$ where $n \in \mathbb{N}^{*}=\mathbb{N} \backslash\{0\}$

Let $M$ be a $\lambda$-term. If, in the tree representation of $M$, there are exactly $n$ abstractors in the minimal path from the root position until the position of some subterm $M_{1}$, then $M_{1}$ is said $\mathbf{n}$-deep in $M$. In other words, $M_{1}$ is in between $n$ abstractors.

Definition 2. We say that $\underline{i}$ occurs as free index in a term $M$ if $i+n$ is $n$-deep in $M$.

Terms like $\left(\left(\left(\left(M_{1} M_{2}\right) M_{3}\right) \ldots\right) M_{n}\right)$ are written as usual $\left(M_{1} M_{2} \ldots M_{n}\right)$. The $\beta$-contraction definition in this notation needs a mechanism which detects and updates free indices of terms. It follows an operator similar to the one presented in [ARKa2001a]. 
Definition 3. Let $M \in \Lambda_{d B}$ and $i \in \mathbb{N}$. The $i$-lift of $M$, denoted as $M^{+i}$, is defined inductively as

$$
\begin{aligned}
& \text { 1. }\left(M_{1} M_{2}\right)^{+i}=\left(M_{1}^{+i} M_{2}^{+i}\right) \\
& \text { 2. }\left(\lambda \cdot M_{1}\right)^{+i}=\lambda \cdot M_{1}^{+(i+1)}
\end{aligned} \quad \text { 3. } \underline{n}^{+i}= \begin{cases}\frac{n+1}{n}, & \text { if } n>i \\
\underline{n}, & \text { if } n \leq i .\end{cases}
$$

The lift of a term $M$ is its 0-lift, denoted as $M^{+}$. Intuitively, the lift of $M$ corresponds to an increment by 1 of all free indices occurring in $M$. Using the i-lift, we are able to present the definition of the substitution used by $\beta$-contractions, similarly to the one presented in [ARKa2001a].

Definition 4. Let $m, n \in \mathbb{N}^{*}$. The $\beta$-substitution for free occurrences of $\underline{n}$ in $M \in \Lambda_{d B}$ by term $N$, denoted as $\{\underline{n} / N\} M$, is defined inductively by

$$
\begin{aligned}
& \text { 1. }\{\underline{n} / N\}\left(M_{1} M_{2}\right)=\left(\{\underline{n} / N\} M_{1}\{\underline{n} / N\} M_{2}\right) \\
& \text { 2. }\{\underline{n} / N\} \lambda \cdot M_{1}=\lambda \cdot\left\{\underline{n+1} / N^{+}\right\} M_{1}
\end{aligned}
$$

Observe that in item 2 of Def. 4 , the lift operator is used to avoid captures of free indices in $N$. We present the $\beta$-contraction as defined in [ARKa2001a].

Definition 5. $\beta$-contraction in $\lambda d B$ is defined by $(\lambda . M N) \rightarrow_{\beta}\{\underline{1} / N\} M$.

Notice that item 3 in Definition 4 , for $n=1$, is the mechanism which does the substitution and updates the free indices in $M$ as consequence of the lead abstractor elimination.

\subsection{The $\lambda s_{e^{-C a l c u l u s}}$}

The $\lambda s_{e}$-calculus is a proper extension of the $\lambda d B$-calculus. Two operators $\sigma$ and $\varphi$ are introduced for substitution and updating, respectively, to control the atomization of the substitution operation by arithmetic constraints.

Definition 6 (Set $\Lambda_{s}$ of $\lambda s_{e}$-terms). The syntax of the $\lambda s_{e}$-calculus, where $n, i, j \in \mathbb{N}^{*}$ and $k \in \mathbb{N}$ is given by

Terms $M::=\underline{n}|(M M)| \lambda . M\left|M \sigma^{i} M\right| \varphi_{k}^{j} M$

The term $M \sigma^{i} N$ represents the term $\{\underline{i} / N\} M$; i.e., the substitution of free occurrences of $\underline{i}$ in $M$ by $N$, updating free variables in $M$ (and in $N$ ). The term $\varphi_{k}^{j} M$ represents $j-1$ applications of the $k$-lift to the term $M$; i.e., $M^{+k^{(j-1)}}$. Table 1 contains the rewriting rules of the $\lambda s_{e}$-calculus together with the rule (Eta), as given in [ARKa2001a].

$=s_{e}$ denotes the equality for the associated substitution calculus, denoted as $s_{e}$, induced by all the rules except ( $\sigma$-generation) and (Eta).

\section{$2.3 \quad$ The $\lambda \sigma$-Calculus}

The $\lambda \sigma$-calculus is given by a first-order rewriting system, which makes substitutions explicit by extending the language with two sorts of objects: terms and substitutions which are called $\lambda \sigma$-expression.

Definition 7 (Set $\Lambda_{\sigma}$ of $\lambda \sigma$-expressions). The $\lambda \sigma$-expressions consist of:

Terms $\quad M::=\underline{1}|(M M)| \lambda \cdot M \mid M[S]$

Substitutions $S::=i \bar{d}|\uparrow| M . S \mid S \circ S$ 


\begin{tabular}{|c|c|c|}
\hline $\begin{array}{l}(\lambda \cdot M N) \\
(\lambda \cdot M) \sigma^{i} N \\
\left(M_{1} M_{2}\right) \sigma^{i} N \\
\underline{n} \sigma^{i} N \\
\varphi_{k}^{i}(\lambda \cdot M) \\
\varphi_{k}^{i}\left(M_{1} M_{2}\right) \\
\varphi_{k}^{i} \underline{n} \\
\left(M_{1} \sigma^{i} M_{2}\right) \sigma^{j} N \\
\left(\varphi_{k}^{i} M\right) \sigma^{j} N \\
\left(\varphi_{k}^{i} M\right) \sigma^{j} N \\
\varphi_{k}^{i}\left(M \sigma^{j} N\right) \\
\varphi_{k}^{i}\left(\varphi_{l}^{j} M\right) \\
\varphi_{k}^{i}\left(\varphi_{l}^{j} M\right) \\
\lambda .(M \underline{1})\end{array}$ & $\begin{aligned} \longrightarrow & M \sigma^{1} N \\
\longrightarrow & \lambda \cdot\left(M \sigma^{i+1} N\right) \\
\longrightarrow & \left(\left(M_{1} \sigma^{i} N\right)\left(M_{2} \sigma^{i} N\right)\right) \\
\longrightarrow & \left\{\begin{array}{l}\frac{n-1}{\varphi_{0}^{i} N} \text { if } n>i \\
\text { if } n=i \\
\underline{n} \quad \text { if } n<i\end{array}\right. \\
\longrightarrow & \lambda .\left(\varphi_{k+1}^{i} M\right) \\
\longrightarrow & \left(\left(\varphi_{k}^{i} M_{1}\right)\left(\varphi_{k}^{i} M_{2}\right)\right) \\
\longrightarrow & \left\{\begin{array}{l}n+i-1 \\
\frac{n}{n} \text { if } n>k \\
\longrightarrow\end{array} \quad\left(M_{1} \sigma^{j+1} N\right) \sigma^{i}\left(M_{2} \sigma^{j-i+1} N\right) \quad \text { if } i \leq j\right. \\
\longrightarrow & \varphi_{k}^{i-1} M \quad \text { if } k<j<k+i \\
\longrightarrow & \varphi_{k}^{i}\left(M \sigma^{j-i+1} N\right) \quad \text { if } k+i \leq j \\
\longrightarrow & \left(\varphi_{k+1}^{i} M\right) \sigma^{j}\left(\varphi_{k+1-j}^{i} N\right) \quad \text { if } j \leq k+1 \\
\longrightarrow & \varphi_{l}^{j}\left(\varphi_{k+1-j}^{i} M\right) \quad \text { if } l+j \leq k \\
\longrightarrow & \varphi_{l}^{j+i-1} M \quad \text { if } l \leq k<l+j \\
\longrightarrow & \text { if } M=s_{e} \varphi_{0}^{2} N\end{aligned}$ & $\begin{array}{l}(\sigma \text {-destruction }) \\
(\varphi \text { - } \lambda \text {-trans. }) \\
(\varphi \text {-app-trans. }) \\
(\varphi \text {-destruction) } \\
(\sigma \text { - } \sigma \text {-trans. }) \\
(\sigma \text { - } \varphi \text {-trans. } 1) \\
(\sigma-\varphi \text {-trans. } 2) \\
(\varphi \text { - } \sigma \text {-trans. }) \\
(\varphi \text { - } \varphi \text {-trans. } 1) \\
(\varphi \text { - } \varphi \text {-trans. } 2) \\
(E \text { ta) }\end{array}$ \\
\hline
\end{tabular}

Table 1. The rewriting system of the $\lambda s_{e}$-calculus with the Eta rule

Substitutions are lists of the form $N / \underline{i}$ indicating that the index $\underline{i}$ should be changed to the term $N$. The expression $i d$ represents a substitution of the form $\{\underline{1} / \underline{1}, \underline{2} / \underline{2}, \ldots\}$ whereas $\uparrow$ is the substitution $\left\{\underline{i+1} / \underline{i} \mid i \in \mathbb{N}^{*}\right\}$. The expression $S \circ S$ represents the composition of substitutions. Moreover, 1 [ $\left.\uparrow^{n}\right]$, where $n \in \mathbb{N}^{*}$, codifies the de Bruijn index $\underline{n+1}$ and $\underline{i}[S]$ represents the value of $\underline{i}$ through the substitution $S$, which can be seen as a function $S(i)$. The substitution M.S has the form $\{M / \underline{1}, S(i) / \underline{i+1}\}$, called the cons of $M$ in $S . M[N . i d]$ starts the simulation of the $\beta$-reduction of $(\lambda . M N)$ in $\lambda \sigma$. Thus, in addition to the substitution of the free occurrences of the index 1 by the corresponding term, free occurrences of indices should be decremented because of the elimination of the abstractor. Table 2 includes the rewriting system of the $\lambda \sigma$-calculus, as presented in [DoHaKi2000].

\begin{tabular}{|lll|}
\hline$(\lambda . M N)$ & $\longrightarrow M[N . i d]$ & $($ Beta $)$ \\
$(M N)[S]$ & $\longrightarrow(M[S] N[S])$ & $($ App $)$ \\
$1[M . S]$ & $\longrightarrow M$ & $($ VarCons $)$ \\
$M[i d]$ & $\longrightarrow M$ & $($ Id) \\
$(\lambda . M)[S]$ & $\longrightarrow \lambda .(M[1 .(S \circ \uparrow)])$ & $($ Abs $)$ \\
$(M[S])[T]$ & $\longrightarrow M[S \circ T]$ & $($ Clos $)$ \\
$i d \circ S$ & $\longrightarrow S$ & $($ IdL) \\
$\uparrow \circ(M . S)$ & $\longrightarrow S$ & $($ ShiftCons $)$ \\
$\left(S_{1} \circ S_{2}\right) \circ S_{3} \longrightarrow S_{1} \circ\left(S_{2} \circ S_{3}\right)$ & $($ AssEnv) \\
$(M . S) \circ T$ & $\longrightarrow M[T] .(S \circ T)$ & $($ MapEnv $)$ \\
$S \circ i d$ & $\longrightarrow S$ & $($ IdR) \\
$1 . \uparrow$ & $\longrightarrow i d$ & $($ VarShift $)$ \\
$1[S] .(\uparrow \circ S)$ & $\longrightarrow S$ & $($ Scons $)$ \\
$\lambda .(M \underline{1})$ & $\longrightarrow N \quad$ if $M={ }_{\sigma} N[\uparrow]$ & Eta) \\
& & \\
\hline
\end{tabular}

Table 2. The rewriting system for the $\lambda \sigma$-calculus with the Eta rule 
This system without (Eta) is equivalent to that of [ACCL91]. The associated substitution calculus, denoted as $\sigma$, is the one induced by all the rules except (Beta) and (Eta), and its equality is denoted as $={ }_{\sigma}$.

\section{The Type Systems}

Definition 8. The syntax of the simple types and contexts is given by:

$$
\text { Types } \tau::=\alpha \mid \tau \rightarrow \tau \quad \text { Contexts } A::=n i l \mid \tau \text {.A }
$$

$\alpha$ ranges over type variables. A type assignment system $\mathcal{S}$ is a set of rules which allows some terms of a given system be associated with a type. A context gives the necessary information used by $\mathcal{S}$ rules to associate a type to a term. In the simply typed $\lambda$-calculus [Hi97], the typable terms are strongly normalizing. The ordered pair $\langle A, \tau\rangle$, of a context and a type, is called a typing in $\mathcal{S}$. For a term $M, A \vdash M: \tau$ denotes that $M$ has type $\tau$ in context $A$, and $\langle A, \tau\rangle$ is called a typing of $M$. Let $\Theta=\langle A, \tau\rangle$ be a typing in $\mathcal{S}$. $\mathcal{S} \Vdash M: \Theta$ denotes that $\Theta$ is a typing of $M$ in $\mathcal{S}$.

The contexts for $\lambda$-terms in de Bruijn notation are sequences of types. Let $A$ be some context and $n \in \mathbb{N}$. Then $A_{<n}$ denotes the first $n-1$ types of $A$. Similarly we define $A_{>n}, A_{\leq n}$ and $A_{\geq n}$. Note that, for $A_{>n}$ and $A_{\geq n}$ the final nil element is included. For $n=0, A_{\leq 0} . A=A_{<0} . A=A$. The length of $A$ is defined as $\mid$ nil $\mid=0$ and, if $A$ is not nil, $|A|=1+\left|A_{>1}\right|$. The addition of some type $\tau$ at the end of a context $A$ is defined as $A . \tau=A_{\leq m} . \tau$.nil, where $|A|=m$.

Given a term $M$, an interesting question is whether it is typable in $\mathcal{S}$ or not. Note that, we are using a Curry-style/implicit typing, where in $\lambda . M$ we did not specify the type of the bound variable ( 1 ). Such terms have many types, depending on the context. Another important question is whether given a term, its so-called most general typing can be found. An answer to this question, which represents any other answer, is called principal typing. Principal typing (which is context independent) is not to be confused with a principal type (which is context dependent). Let $\Theta$ be a typing in $\mathcal{S}$ and $\operatorname{Terms}_{\mathcal{S}}(\Theta)=\{M \mid \mathcal{S} \Vdash M: \Theta\}$. J.B. Wells introduced in [We2002] a system-independent definition of PT and proved that it generalizes previous system-specific definitions.

Definition 9 ( [We2002]). A typing $\Theta$ in system $\mathcal{S}$ is principal for some term $M$ if $\mathcal{S} \Vdash M: \Theta$ and for any $\Theta^{\prime}$ such that $\mathcal{S} \Vdash M: \Theta^{\prime}$ we have that $\Theta \leq_{\mathcal{S}} \Theta^{\prime}$, where $\Theta_{1} \leq_{\mathcal{S}} \Theta_{2} \Longleftrightarrow \operatorname{Terms}_{\mathcal{S}}\left(\Theta_{1}\right) \subseteq \operatorname{Terms}_{\mathcal{S}}\left(\Theta_{2}\right)$.

In simply typed systems the principal typing notion is tied to type substitution and weakening. Weakening allows one to add unnecessary information to contexts. Type substitution maps type variables to types. Given a type substitution $s$, the extension for functional types is straightforward as $s(\sigma \rightarrow \tau)=s(\sigma) \rightarrow s(\tau)$ and the extension for sequential contexts as $s(n i l)=n i l$ and $s(\tau . A)=s(\tau) . s(A)$. The extension for typings is given by $s(\Theta)=\langle s(A), s(\tau)\rangle$. 


\subsection{Principal typings for the simply typed $\lambda$-calculus in de Bruijn notation $T A_{\lambda d B}$}

Definition 10. (The System $T A_{\lambda d B}$ ) The $T A_{\lambda d B}$ typing rules are given by

$$
\begin{array}{lccc}
\text { (Var) } & \tau . A \vdash \underline{1}: \tau & (\text { Varn }) & \frac{A \vdash \underline{n}: \tau}{\sigma \cdot A \vdash \underline{n+1}: \tau} \\
(\text { Lambda) } & \frac{\sigma \cdot A \vdash M: \tau}{A \vdash \lambda \cdot M: \sigma \rightarrow \tau} & \text { (App) } & \frac{A \vdash M: \sigma \rightarrow \tau \quad A \vdash N: \sigma}{A \vdash(M N): \tau}
\end{array}
$$

This system is similar to $T A_{\lambda}$ ( [Hi97]). The rule (Varn) allows the construction of contexts as sequences.

Lemma 1. Let $M$ be a $\lambda d B$-term. If $A \vdash M: \tau$, then $A . \sigma \vdash M: \tau$. Hence, the rule $\frac{A \vdash M: \tau}{A . \sigma \vdash M: \tau}(\lambda d B$-weak $)$ holds in the system $T A_{\lambda d B}$.

Using ( $\lambda d B$-weak) and type substitution, we follow the definition of [We2002] for Hindley's Principal Typing to define principal typing for the $\lambda d B$-calculus.

Definition 11. A principal typing in $T A_{\lambda d B}$ of a term $M$ is the typing $\Theta=$ $\langle A, \tau\rangle$ such that

1. $T A_{\lambda d B} \Vdash M: \Theta$

2. If $T A_{\lambda d B} \Vdash M: \Theta^{\prime}$ for any typing $\Theta^{\prime}=\left\langle A^{\prime}, \tau^{\prime}\right\rangle$, then there exists some substitution s such that $s(A)=A_{\leq|A|}^{\prime}$. nil and $s(\tau)=\tau^{\prime}$.

Observe that, given a principal typing $\langle A, \tau\rangle$ of $M$, the context $A$ is the shortest context where $M$ can be typable. In contrast to the $\lambda$-calculus with names, where the context from a principal typing of $M$ is the smallest set because it declares types for exactly the free variables of $M$, the context from a principal typing in $\lambda d B$ may has some type declaration for variables not occurring in the term, to maintain the ordered structure of contexts. For example, a PT for $\underline{2}$ is $\left\langle\tau_{1} . \tau_{2} . n i l, \tau_{2}\right\rangle$.

As is the case for the simply typed $\lambda$-calculus with names, the best way to assure that Definition 11 is the correct translation of the PT concept, is to verify that Definition 11 corresponds to Definition 9.

Theorem 1. A typing $\Theta$ is principal in $T A_{\lambda d B}$ according to Definition 11 iff $\Theta$ is principal in $T A_{\lambda d B}$ according to Definition 9.

We now present a type inference algorithm for $\lambda d B$-terms, similarly to the one in [AyMu2000] for $\lambda s_{e}$, to verify whether $T A_{\lambda d B}$ has PT according to Definition 11 . Given any term $M$, decorate each subterm with a new type variable as subscript and a new context variable as superscript, obtaining a new term denoted as $M^{\prime}$. For example, for term $\lambda .(\underline{2} \underline{1})$ we have the decorated term $\left(\lambda .\left(\underline{2} \tau_{\tau_{1}}^{A_{1}} \underline{1}_{\tau_{2}}^{A_{2}}\right)_{\tau_{3}}^{A_{3}}\right)_{\tau_{4}}^{A_{4}}$. Then, rules from Table 3 are applied to pairs of the form $\langle\langle R, E\rangle\rangle$, where $\mathrm{R}$ is a set of decorated terms and $\mathrm{E}$ a set of equations on type and context variables.

The inference rules in Table 3 are given according to the typing rules of $T A_{\lambda d B}$. Type inference for $M$ starts with $\left\langle\left\langle R_{0}, \varnothing\right\rangle\right\rangle$, where $R_{0}$ is the set of all $M^{\prime}$ subterms. The rules from Table 3 are applied until one reaches $\left\langle\left\langle\varnothing, E_{f}\right\rangle\right\rangle$, where $E_{f}$ is a set of first-order equations over context and type variables. 


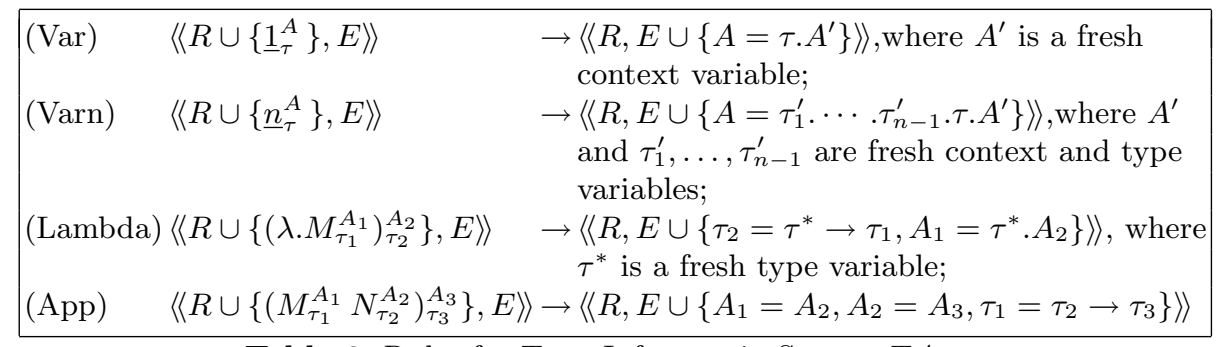

Table 3. Rules for Type Inference in System $T A_{\lambda d B}$

Example 1. Let $M=\lambda .(\underline{2} \underline{1})$. Then $M^{\prime}=\left(\lambda .\left(\underline{2}_{\tau_{1}}^{A_{1}} \underline{1}_{\tau_{2}}^{A_{2}}\right)_{\tau_{3}}^{A_{3}}\right)_{\tau_{4}}^{A_{4}}$ and $R_{0}=\left\{\underline{2}{ }_{\tau_{1}}^{A_{1}}\right.$, $\left.\underline{1}_{\tau_{2}}^{A_{2}},\left(\underline{2}_{\tau_{1}}^{A_{1}} \underline{1}_{\tau_{2}}^{A_{2}}\right)_{\tau_{3}}^{A_{3}},\left(\lambda .\left(\underline{2}_{\tau_{1}} \underline{1}_{\tau_{2}}^{A_{2}}\right)_{\tau_{3}}^{A_{3}}\right)_{\tau_{4}}^{A_{4}}\right\}$. Using the rules in Table 3 we have the following reduction:

$$
\begin{aligned}
& \left\langle\left\langle R_{0}, \emptyset\right\rangle\right\rangle \rightarrow \text { Varn } \\
& \left\langle\left\langle R_{1}=R_{0} \backslash\left\{\underline{2}_{\tau_{1}}^{A_{1}}\right\}, E_{1}=\left\{A_{1}=\tau_{1}^{\prime} \cdot \tau_{1} \cdot A_{1}^{\prime}\right\}\right\rangle \rightarrow \rightarrow_{\text {Var }}\right. \\
& \left\langle\left\langle R_{2}=R_{1} \backslash\left\{\underline{1}_{\tau_{2}}^{A_{2}}\right\}, E_{2}=E_{1} \cup\left\{A_{2}=\tau_{2} \cdot A_{2}^{\prime}\right\}\right\rangle\right\rangle \rightarrow \text { App } \\
& \left\langle\left\langle R_{3}=R_{2} \backslash\left\{\left(\underline{2}_{\tau_{1}}^{A_{1}} \underline{1}_{\tau_{2}}^{A_{2}}\right)_{\tau_{3}}^{A_{3}}\right\}, E_{3}=E_{2} \cup\left\{A_{1}=A_{2}, A_{2}=A_{3}, \tau_{1}=\tau_{2} \rightarrow \tau_{3}\right\}\right\rangle\right\rangle \rightarrow \text { Lambda } \\
& \left\langle\left\langle\emptyset=R_{3} \backslash\left\{\left(\lambda .\left(\underline{2}_{\tau_{1}}^{A_{1}} \underline{1}_{\tau_{2}}^{A_{2}}\right)_{\tau_{3}}^{A_{3}}\right)_{\tau_{4}}^{A_{4}}\right\}, E_{4}=E_{3} \cup\left\{\tau_{4}=\tau_{1}^{*} \rightarrow \tau_{3}, A_{3}=\tau_{1}^{*} \cdot A_{4}\right\}\right\rangle\right\rangle
\end{aligned}
$$

Thus, $E_{4}=E_{f}$. Solving the trivial equation over context variables, i.e. $A_{1}=$ $A_{2}=A_{3}$, and using variables of smaller subscripts, one gets $\left\{\tau_{1}=\tau_{2} \rightarrow \tau_{3}, \tau_{4}=\right.$ $\left.\tau_{1}^{*} \rightarrow \tau_{3}, A_{1}=\tau_{1}^{\prime} \cdot \tau_{1} \cdot A_{1}^{\prime}, A_{1}=\tau_{2} \cdot A_{2}^{\prime}, A_{1}=\tau_{1}^{*} \cdot A_{4}\right\}$. Thus, simplifying one gets $\left\{\tau_{1}=\right.$ $\left.\tau_{2} \rightarrow \tau_{3}, \tau_{4}=\tau_{1}^{*} \rightarrow \tau_{3}, \tau_{1}^{\prime} \cdot \tau_{1} \cdot A_{1}^{\prime}=\tau_{2} \cdot A_{2}^{\prime}=\tau_{1}^{*} . A_{4}\right\}$. From these equations one gets the most general unifier (mgu for short) $\tau_{4}=\tau_{2} \rightarrow \tau_{3}$ and $A_{4}=\left(\tau_{2} \rightarrow \tau_{3}\right)$. $A_{1}^{\prime}$, for the variables of interest. Since the context must be the shortest one, $A_{1}^{\prime}=n i l$ and $\left\langle\left(\tau_{2} \rightarrow \tau_{3}\right) . n i l, \tau_{2} \rightarrow \tau_{3}\right\rangle$ is a principal typing of $M$.

From Definition 11 and by the uniqueness of the solutions of the type inference algorithm, one deduces that $T A_{\lambda d B}$ satisfies PT. The next theorem says that every typable term has a principal typing.

Theorem 2 (Principal Typings for $T A_{\lambda d B}$ ). $T A_{\lambda d B}$ satisfies the property of having principal typings.

\subsection{Principal typings for $T A_{\lambda s_{e}}$, the simply typed $\lambda s_{e}$}

The typed version of $\lambda s_{e}$ presented is in Curry style, which we have verified to have the same properties which properties as the version in Church style presented in [ARKa2001a]. In particular, the properties in question being: weak normalisation (WN), confluence (CR) and subject reduction (SR). Thus, the syntax of $\lambda s_{e}$-terms and the rules are the same as the untyped version.

Since the syntax of $\lambda s_{e}$ remains close to the $\lambda d B$-calculus, to have a type assignment system for the $\lambda s_{e}$-calculus we only need to add typing rules to $T A_{\lambda d B}$ for the two new kinds of terms.

Definition 12 (The System $T A_{\lambda s_{e}}$ ). $T A_{\lambda s_{e}}$ is given by (Var), (Varn), (App), (Lambda) from Definiton 10 and the following new rules.

$$
\text { (Sigma) } \frac{A_{\geq i} \vdash N: \rho \quad A_{<i} \cdot \rho . A_{\geq i} \vdash M: \tau}{A \vdash M \sigma^{i} N: \tau} \quad \text { (Phi) } \frac{A_{\leq k} \cdot A_{\geq k+i} \vdash M: \tau}{A \vdash \varphi_{k}^{i} M: \tau}
$$


Weakening for $\lambda s_{e}$ is done the same way as for $\lambda d B$, adding types at the end of a context, giving the following lemma.

Lemma 2 (Weakening for $\left.\lambda s_{e}\right)$. The rule $\left(\lambda s_{e}\right.$-weak) holds in System $T A_{\lambda s_{e}}$, where $\frac{A \vdash M: \tau}{A . \sigma \vdash M: \tau}\left(\lambda s_{e}-w e a k\right)$.

Consequently, the definition of principal typings in $\lambda s_{e}$ is the same as that for $T A_{\lambda d B}$. For the sake of completeness we repeat it here.

Definition 13 (Principal Typings in $T A_{\lambda s_{e}}$ ). A principal typing of a term $M$ in $T A_{\lambda s_{e}}$ is a typing $\Theta=\langle A, \tau\rangle$ such that

1. $T A_{\lambda s_{e}} \Vdash M: \Theta$

2. If $T A_{\lambda s_{e}} \Vdash M: \Theta^{\prime}$ for any typing $\Theta^{\prime}=\left\langle A^{\prime}, \tau^{\prime}\right\rangle$, then there exists a substitution $s$ such that $s(A)=A_{\leq|A|}^{\prime}$. nil and $s(\tau)=\tau^{\prime}$.

Theorem 3. A typing $\Theta$ is principal in $T A_{\lambda s_{e}}$ according to Definition 13 iff $\Theta$ is principal in $T A_{\lambda s_{e}}$ according to Definition 9.

We now present a type inference algorithm for the $\lambda s_{e}$-calculus, similarly to that of [AyMu2000]. The algorithm is composed of the rules from Table 3 and the new rules in Table 4.

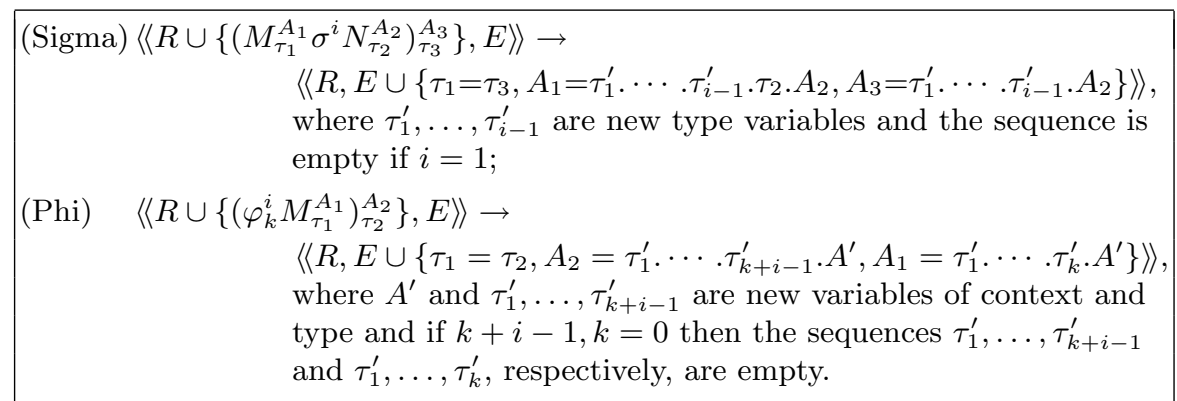

Table 4. Type inference rules for the $\lambda s_{e}$-Calculus

Similarly to the previous algorithm, the rules of the Table 4 were developed according to the rules of Definition 12. The decorated term associated with $M$, denoted as $M^{\prime}$, has a syntax close to the one of decorated $\lambda d B$-terms: any subterm is decorated with its type and its context variables. The rules are applied to pairs $\langle\langle R, E\rangle\rangle$, starting from the pair $\left\langle\left\langle R_{0}, \varnothing\right\rangle\right\rangle$, as was done to $T A_{\lambda d B}$.

Example 2. For the $\lambda s_{e}$-term $M=\lambda .\left(\left(\underline{1} \sigma^{2} \underline{2}\right)\left(\varphi_{0}^{2} \underline{2}\right)\right)$, one obtains the corresponding $R_{0}$ from $M^{\prime}=\left(\lambda .\left(\left(\underline{1}_{\tau_{1}}^{A_{1}} \sigma^{2} \underline{2}_{\tau_{2}}^{A_{2}}\right)_{\tau_{3}}^{A_{3}}\left(\varphi_{0}^{2} \underline{2}_{\tau_{4}}^{A_{4}}\right)_{\tau_{5}}^{A_{5}}\right)_{\tau_{6}}^{A_{6}}\right)_{\tau_{7}}^{A_{7}}$. Then, applying the rules in Table 3 and 4 to the pair $\left\langle\left\langle R_{0}, \emptyset\right\rangle\right\rangle$, obtaining the pair $\left\langle\left\langle\emptyset, E_{f}\right\rangle\right\rangle$, and simplifying $E_{f}$, similarly to the example 1 , one obtains the system of equations $\left\{\tau_{1}=\tau_{4} \rightarrow \tau_{6}, \tau_{7}=\tau_{1}^{*} \rightarrow \tau_{6}, \tau_{1} \cdot A_{1}^{\prime}=\tau_{2}^{\prime} \cdot \tau_{2} \cdot A_{2}, \tau_{2}^{\prime} \cdot A_{2}=\tau_{4}^{\prime} \cdot \tau_{3}^{\prime} \cdot \tau_{4} \cdot A_{3}^{\prime}=\tau_{1}^{*} \cdot A_{7}, A_{2}=\right.$ $\left.\tau_{1}^{\prime} \cdot \tau_{2} . A_{2}^{\prime}\right\}$ from which one has the mgu $\tau_{7}=\left(\tau_{2} \rightarrow \tau_{6}\right) \rightarrow \tau_{6}$ and $A_{7}=\tau_{1}^{\prime} \cdot \tau_{2} \cdot A_{2}^{\prime}$ for variables of interest.

Theorem 4 (Principal Typings for $T A_{\lambda s_{e}}$ ). $T A_{\lambda s_{e}}$ satisfies the property of having principal typings. 


\subsection{Principal typings for $T A_{\lambda \sigma}$, the simply typed $\lambda \sigma$}

The typing rules of the $\lambda \sigma$-calculus provide types for objects of sort term as well as for objects of sort substitution. An object of sort substitution, due to its semantics, can be viewed as a list of terms. Consequently, its type is a context. $S \triangleright A$ denotes that the object of sort substitution $S$ has type $A$.

Definition 14 (The System $T A_{\lambda \sigma}$ ). $T A_{\lambda \sigma}$ is given by the following typing rules.

$$
\begin{aligned}
& \text { (var) } \quad \tau . A \vdash \underline{1}: \tau \quad \text { (lambda) } \frac{\sigma . A \vdash M: \tau}{A \vdash \lambda . M: \sigma \rightarrow \tau}
\end{aligned}
$$

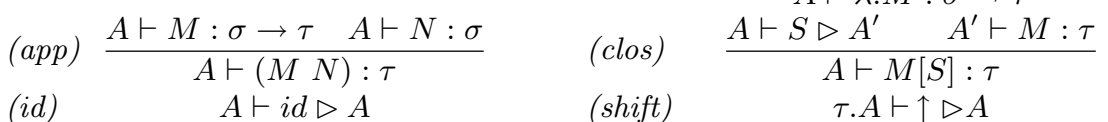

$$
\begin{aligned}
& \text { (cons) } \frac{A \vdash M: \tau \quad A \vdash S \triangleright A^{\prime}}{A \vdash M . S \triangleright \tau . A^{\prime}} \quad \text { (comp) } \frac{A \vdash S \triangleright A^{\prime \prime} \quad A^{\prime \prime} \vdash S^{\prime} \triangleright A^{\prime}}{A \vdash S^{\prime} \circ S \triangleright A^{\prime}}
\end{aligned}
$$

Observe that the name of the typing rules begin with lower-case letters, while the rewriting rules with upper-case letters. As for $\lambda s_{e}$, the typed version of the $\lambda \sigma$-calculus is presented in Curry style. We have verified that the Curry style version has WN, CR and SR as the Church style version of [DoHaKi2000].

For $T A_{\lambda \sigma}$ the notion of typing has to be adapted since the $\lambda \sigma$-expression of sort substitution is decorated with contexts variables as types and as contexts. Thus, one may say that $\Theta=\langle A, \mathbb{T}\rangle$ is a typing of a $\lambda \sigma$-expression in $T A_{\lambda \sigma}$, where $\mathbb{T}$ can be either a type or a context. If the analysed expression belongs to the $\lambda$-calculus, the notion of typing corresponds to that of $T A_{\lambda d B}$.

Lemma 3 (Weakening for $\lambda \sigma$ ). Let $M$ be a $\lambda \sigma$-term and $S$ a $\lambda \sigma$-substitution. If $A \vdash M: \tau$, then A. $\sigma \vdash M: \tau$, for any type $\sigma$. Similarly, if $A \vdash S \triangleright A^{\prime}$, then A. $\sigma \vdash S \triangleright A^{\prime} . \sigma$. Hence, the rules $(\lambda \sigma$-tweak) and $(\lambda \sigma$-sweak $)$ hold in System $T A_{\lambda \sigma}$, where

$$
\frac{A \vdash M: \tau}{A . \sigma \vdash M: \tau}(\lambda \sigma \text {-tweak }) \quad \frac{A \vdash S \triangleright A^{\prime}}{A . \sigma \vdash S \triangleright A^{\prime} . \sigma}(\lambda \sigma \text {-sweak })
$$

Lemma 3 and type substitution allow us present a definition for PT in $T A_{\lambda \sigma}$.

Definition 15 (Principal Typings in $T A_{\lambda \sigma}$ ). A principal typing of an expression $M$ in $T A_{\lambda \sigma}$ is a typing $\Theta=\langle A, \mathbb{T}\rangle$ such that

1. $T A_{\lambda \sigma} \Vdash M: \Theta$

2. If $T A_{\lambda \sigma} \Vdash M: \Theta^{\prime}$ for any typing $\Theta^{\prime}=\left\langle A^{\prime}, \mathbb{T}^{\prime}\right\rangle$, then there exists a substitution s such that $s(A)=A_{\leq|A|}^{\prime}$. nil and if $\mathbb{T}$ is a type, $s(\mathbb{T})=\mathbb{T}^{\prime}$, otherwise we have that $s(\mathbb{T})=\mathbb{T}_{\leq|\mathbb{T}|}^{\prime}$. nil.

We might verify if this PT definition has a correspondence with Wells' systemindependent definition [We2002].

Theorem 5. A typing $\Theta$ is principal in $T A_{\lambda \sigma}$ according to Definition 15 iff $\Theta$ is principal in $T A_{\lambda \sigma}$ according to Definition 9.

We now present an algorithm for type inference, to verify if $\mathrm{TA}_{\lambda \sigma}$ has $\mathrm{PT}$ according to Definition 15. Thus, given an expression $M$, we will work with the decorated expression $M^{\prime}$ but the type for substitutions is a context as well. We use the same syntax for decorated expressions as in [Bo95]. 


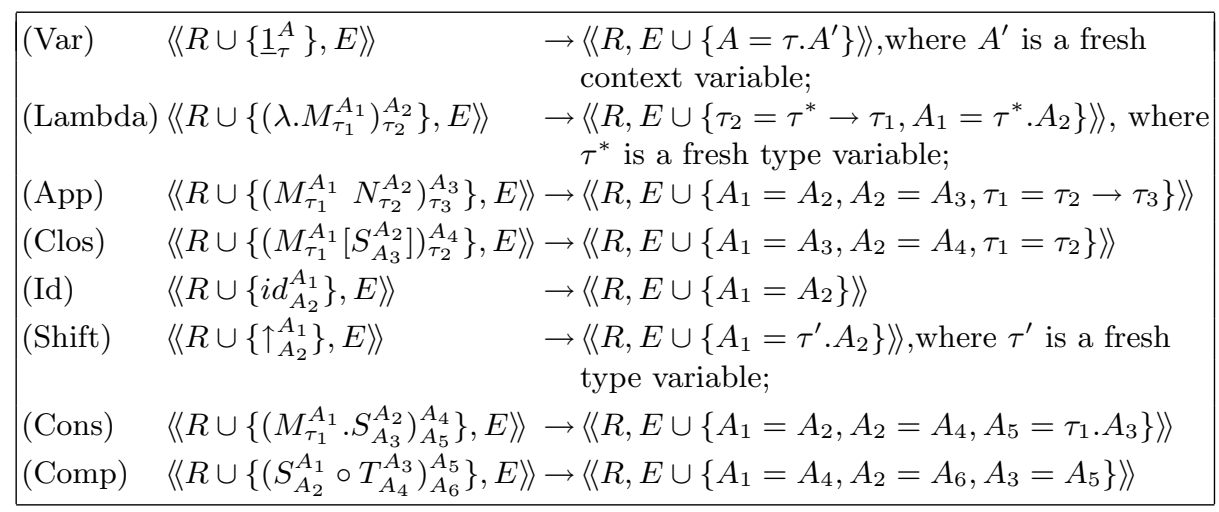

Table 5. Type inference rules for the $\lambda \sigma$-calculus

The inference rules presented in Table 5 are given according to the typing rules of the system $T A_{\lambda \sigma}$ presented in Definition 14. Similarly to the previous algorithm, the rules are applied to pairs $\langle\langle R, E\rangle\rangle$, where $R$ is a set of subexpressions of $M^{\prime}$ and $E$ a set of equations over type and context variables.

Example 3. For $M=(\underline{2} . i d) \circ \uparrow$ one has $M^{\prime}=\left(\left(\left(\underline{1}_{\tau_{1}}^{A_{1}}\left[\uparrow_{A_{3}}^{A_{2}}\right]\right)_{\tau_{2}}^{A_{4}} \cdot i d_{A_{6}}^{A_{5}}\right)_{A_{8}}^{A_{7}} \circ \uparrow_{A_{10}}^{A_{9}}\right)_{A_{12}}^{A_{11}}$. Then $R_{0}=\left\{\left(\underline{1}_{\tau_{1}}^{A_{1}}\left[\uparrow_{A_{3}}^{A_{2}}\right]\right)_{\tau_{2}}^{A_{4}},\left(\left(\underline{1}_{\tau_{1}}^{A_{1}}\left[\uparrow_{A_{3}}^{A_{2}}\right]\right)_{\tau_{2}}^{A_{4}} \cdot i d_{A_{6}}^{A_{5}}\right)_{A_{8}}^{A_{7}},\left(\left(\left(\underline{1}_{\tau_{1}}^{A_{1}}\left[\uparrow_{A_{3}}^{A_{2}}\right]\right)_{\tau_{2}}^{A_{4}} \cdot i d_{A_{6}}^{A_{5}}\right)_{A_{8}}^{A_{7}} \circ \uparrow_{A_{10}}^{A_{9}}\right)_{A_{12}}^{A_{11}}\right.$, $\left.\underline{1}_{\tau_{1}}^{A_{1}}, \uparrow_{A_{3}}^{A_{2}}, i d_{A_{6}}^{A_{5}}, \uparrow_{A_{10}}^{A_{9}}\right\}$. Applying the rules from Table 5 to the pair $\left\langle\left\langle R_{0}, \emptyset\right\rangle\right\rangle$ until obtain the pair $\left\langle\left\langle\emptyset, E_{f}\right\rangle\right\rangle$ and simplifying $E_{f}$, as in example 1, one obtains the set of equations $\left\{\tau_{1}=\tau_{2}, A_{11}=A_{12}=\tau_{2} . A_{2}, A_{2}=\tau_{1}^{\prime} . A_{1}, A_{1}=\tau_{1} . A_{1}^{\prime}\right\}$. From this equational system one obtains the mgu $A_{11}=A_{12}=\tau_{1} \cdot \tau_{1}^{\prime} \cdot \tau_{1} \cdot A_{1}^{\prime}$, for the variables of interest. Thus, $\left\langle\tau_{1} \cdot \tau_{1}^{\prime} \cdot \tau_{1} \cdot n i l, \tau_{1} \cdot \tau_{1}^{\prime} \cdot \tau_{1} \cdot n i l\right\rangle$ is a principal typing of $M$.

Theorem 6 (Principal Typings for $T A_{\lambda \sigma}$ ). $T A_{\lambda \sigma}$ satisfies the property of having principal typings.

\section{Conclusions and Future Work}

We considered for $\lambda s_{e}$ and $\lambda \sigma$ particular notions of principal typings and gave respective definitions which we proved to agree with the system-independent notion of Wells in [We2002]. The adaptation of this general notion of principal typings for the $\lambda \sigma$ requires special attention, since this calculus enlarges the language of the $\lambda$-calculus by introducing a new sort of substitution objects, whose types are contexts. Thus, the provided PT notion has to deal with the principality of substitution objects as well. Then, the property of having principal typings is straightforwardly proved by revisiting type inference algorithms for the $\lambda s_{e}$ and the $\lambda \sigma$, previously presented in [AyMu2000] and [Bo95], respectively. The result is based on the correctness, completeness and uniqueness of solutions given by adequate first-order unification algorithms (e.g. see the unification algorithm given in [Hi97]).

The investigation of this property for more elaborated typing systems of explicit substitutions is an interesting work to be done. 


\section{References}

[ACCL91] M. Abadi, L. Cardelli, P.-L. Curien, and J.-J. Lévy. Explicit Substitutions. J. of Functional Programming, 1(4):375-416, 1991.

[ARMoKa2005] M. Ayala-Rincón, F. de Moura, and F. Kamareddine. Comparing and Implementing Calculi of Explicit Substitutions with Eta-Reduction. Annals of Pure and Applied Logic, 134:5-41, 2005.

[ARKa2001a] M. Ayala-Rincón and F. Kamareddine. Unification via the $\lambda s_{e}$-Style of Explicit Substitution. The Logical Journal of the Interest Group in Pure and Applied Logics, 9(4):489-523, 2001.

[AyMu2000] M. Ayala-Rincón and C. Muñoz. Explicit Substitutions and All That. Revista Colombiana de Computación, 1(1):47-71, 2000.

[Bo95] P. Borovanský. Implementation of Higher-Order Unification Based on Calculus of Explicit Substitutions. In M. Bartošek, J. Staudek, and J. Wiedermann, editors, Proceedings of the SOFSEM'95: Theory and Practice of Informatics, volume 1012 of $L N C S$, pages 363-368. Springer Verlag, 1995.

[deBru72] N. G. de Bruijn. Lambda calculus notation with nameless dummies, a tool for automatic formula manipulation, with application to the Church-Rosser theorem. Indagationes Mathematicae, 34:381-392, 1972.

[DoHaKi2000] G. Dowek, T. Hardin, and C. Kirchner. Higher-order Unification via Explicit Substitutions. Information and Computation, 157(1/2):183-235, 2000.

[Hi97] J. R. Hindley. Basic Simple Type Theory. Number 42 in Cambridge Tracts in Theoretical Computer Science. Cambridge University Press, 1997.

[KR97] F. Kamareddine and A. Ríos. Extending a $\lambda$-calculus with Explicit Substitution which Preserves Strong Normalisation into a Confluent Calculus on Open Terms. J. of Func. Programming, 7:395-420, 1997.

[Mel95] P.-A. Melliès. Typed $\lambda$-calculi with explicit substitutions may not terminate. In Proc. of TLCA'95, volume 902 of LNCS, pages 328-334. Springer Verlag, 1995.

[NaWi98] G. Nadathur and D. S. Wilson. A Notation for Lambda Terms A Generalization of Environments. Theoretical Computer Science, 198:49-98, 1998.

[We2002] J. Wells. The essence of principal typings. In Proc. 29th International Colloquium on Automata, Languages and Programming, ICALP 2002, volume 2380 of LNCS, pages 913-925. Springer Verlag, 2002. 


\section{A Proofs}

The proofs are divided in three parts: A.1, where the proof of weakening for each type system is given; A.2, where the three proofs of the correspondence between system-independent and system-specific definition of PT are merged in one; and A.3, where the three proofs of PT are also merged.

\section{A.1 Proofs of weakening}

Proof (Lemma 1 (Weakening for $\left.T A_{\lambda d B}\right)$ ).

Let $A \vdash M: \tau$. We will prove a more general result stating, for $i \in \mathbb{N}$, that $A_{\leq i} . \sigma \cdot A_{>i} \vdash M^{+i}: \tau$. The proof is done by induction on the structure of $M$. Note that if $i \geq m$, where $m=|A|$, then $\sigma$ is added at the end of $A$.

1) $M=\underline{n}$ : Suppose $A \vdash \underline{n}: \tau$. If $n \leq i$, then $\underline{n}^{+i}=\underline{n}$. The $\sigma$ addition at the $i+1$-th position changes only types of indices $\geq i+1$, thus one has trivially that $A_{\leq i} . \sigma . A_{>i} \vdash \underline{n}: \tau$. If $n>i$, then $\underline{n}^{+i}=\underline{n+1}$. By (Varn) $i$ times one has $A_{>i} \vdash \underline{n-i}: \tau$. Thus, by (Varn) applied $i+1$ times, one has that $A_{\leq i} . \sigma . A_{>i} \vdash \underline{n+1}: \tau$.

2) $M=\left(M_{1} M_{2}\right)$ : Suppose $A \vdash\left(M_{1} M_{2}\right): \tau$. By $(A p p), A \vdash M_{1}: \rho \rightarrow \tau$ and $A \vdash M_{2}: \rho$. By induction hypothesis (IH), $A_{\leq i} . \sigma . A_{>i} \vdash M_{1}^{+i}: \rho \rightarrow \tau$ and $A_{\leq i} . \sigma \cdot A_{>i} \vdash M_{2}^{+i}: \rho$. Thus, by $(A p p), A_{\leq i} . \sigma \cdot \bar{A}_{>i} \vdash\left(M_{1}^{+i} M_{2}^{+i}\right): \tau$.

3) $\bar{M}=\lambda . N$ : Suppose $A \vdash \lambda . N: \tau$. By $(L \bar{a}$ b bda) one has that $\rho . A \vdash N: \mu$, where $\tau=\rho \rightarrow \mu$. By IH one has $\rho . A_{\leq i} \cdot \sigma \cdot A_{>i} \vdash N^{+(i+1)}: \mu$. Thus, by $($ Lambda $), A_{\leq i} . \sigma . A_{>i} \vdash \lambda . N^{+(i+1)}: \rho \rightarrow \mu=\tau$.

Since all the information about $M$ free indices is in context $A$, one has that a maximum value for a free index occurrence, at 0-deep in $M$, is $m=|A|$. Consequently, $M^{+j}=M$ for any $j \geq m$. Thus, for $i=m$, we have that $A . \sigma \vdash$ $M: \tau$, for any type $\sigma$. Then a weak rule for $T A_{\lambda d B}$ is admissible, adding types at the end of the context. A type addition in any other position of context $A$ would require updating some free indices, then $M^{+i}$ would correspond to a different function from the one to which the term $M$ corresponds.

Proof (Lemma 2 (Weakening for $\left.T A_{\lambda s_{e}}\right)$ ). Induction on the structure of $M$.

1) $M=\underline{n}$ : Let $A \vdash \underline{n}: \tau$. Since the type addition at the end of $A$ does not change any free index type, one has trivially that $A . \sigma \vdash \underline{n}: \tau$.

2) $M=\left(M_{1} M_{2}\right)$ : Let $A \vdash\left(M_{1} M_{2}\right): \tau$. By $(A p p), A \vdash M_{1}: \rho \rightarrow \tau$ and $A \vdash M_{2}: \rho$, for some $\rho$. By IH, $A . \sigma \vdash M_{1}: \rho \rightarrow \tau$ and $A . \sigma \vdash M_{2}: \rho$. Thus, by $(A p p), A . \sigma \vdash\left(M_{1} M_{2}\right): \tau$.

3) $M=\lambda . N$ : Let $A \vdash \lambda . N: \tau$. By $(L a m b d a), \rho . A \vdash N: \mu$, where $\tau=\rho \rightarrow \mu$. By IH, $\rho . A . \sigma \vdash N: \mu$. Thus, by (Lambda), A. $\sigma \vdash \lambda . N: \tau$.

4) $M=M_{1} \sigma^{i} M_{2}$ : Let $A \vdash M_{1} \sigma^{i} M_{2}: \tau$. By (Sigma), $A_{\geq i} \vdash M_{2}: \rho$ and $A_{<i} . \rho . A_{\geq i} \vdash M_{1}: \tau$. By IH, $A_{\geq i} . \sigma \vdash M_{2}: \rho$ and $A_{<i} . \rho . A_{\geq i} . \sigma \vdash M_{1}: \tau$. Thus, by (Sigma), A. $\sigma \vdash M_{1} \sigma^{i} M_{2}: \tau$.

5) $M=\varphi_{k}^{i} N$ : Let $A \vdash \varphi_{k}^{i} N: \tau$. By $(P h i), A_{\leq k} \cdot A_{\geq k+i} \vdash N: \tau$. By IH, $A_{\leq k} \cdot A_{\geq k+i} . \sigma \vdash N: \tau$. Thus, by $(P h i), A . \sigma \vdash \varphi_{k}^{i} N: \tau$. 
The proof of Lemma 3 needs some auxiliary definitions and lemmas.

Definition 16. Let $M$ be a $\lambda \sigma$-expression. Define $\|\cdot\|: \Lambda_{\sigma} \rightarrow \mathbb{N}$ as

$$
\begin{aligned}
\|(M N)\| & =\|M\|+\|N\| & \|\underline{1}\| & =0 \\
\|\lambda . M\| & =\|M\| & \|i d\| & =0 \\
\|M[S]\| & =\|M\|+\|S\| & \|\uparrow\| & =0 \\
\|S \circ T\| & =\|S\|+\|T\| & \|M . S\| & =1+\|M\|+\|S\|
\end{aligned}
$$

Lemma 4. In $\lambda \sigma$, if $\|S\|=0$ and $A \vdash S \triangleright A^{\prime}$, then $A . \sigma \vdash S \triangleright A^{\prime} . \sigma$.

Proof. By induction on the structure of $S$ where $\|S\|=0$.

1) $S=i d$ : By (id) one has $A . \sigma \vdash i d \triangleright A^{\prime} . \sigma$, trivially.

2) $S=\uparrow$ : Let $A \vdash \uparrow \triangleright A^{\prime}$ where, by (shift), $A=\tau$. $A^{\prime}$. Thus $A . \sigma \vdash \uparrow \triangleright A^{\prime} . \sigma$.

3) $S=S_{1} \circ S_{2}$ : Let $A \vdash S_{1} \circ S_{2} \triangleright A^{\prime}$. By (comp), one has that $A \vdash S_{2} \triangleright A^{\prime \prime}$ and $A^{\prime \prime} \vdash S_{1} \triangleright A^{\prime}$, for some $A^{\prime \prime}$. By IH one has $A . \sigma \vdash S_{2} \triangleright A^{\prime \prime} . \sigma$ and $A^{\prime \prime} . \sigma \vdash S_{1} \triangleright A^{\prime} . \tau$. Thus, by (comp), A. $\sigma \vdash S_{1} \circ S_{2} \triangleright A^{\prime} . \sigma$.

Lemma 5. In $\lambda \sigma$, if $\|M\|=0$ and $A \vdash M: \tau$, then $A . \sigma \vdash M: \tau$.

Proof. By induction on on the structure of $M$ where $\|M\|=0$.

1) $M=\underline{1}$ : Let $A \vdash \underline{1}: \tau$. By (var) one has that $A=\tau$. $A^{\prime}$, for some $A^{\prime}$. Thus one has $A . \sigma \vdash \underline{1}: \tau$, trivially.

2) $M=\left(M_{1} M_{2}\right)$ : Let $A \vdash\left(M_{1} M_{2}\right): \tau$. By (app), $A \vdash M_{1}: \rho \rightarrow \tau$ and $A \vdash M_{2}: \rho$, for some $\rho$. By IH, $A . \sigma \vdash M_{1}: \rho \rightarrow \tau$ and $A . \sigma \vdash M_{2}: \rho$. Thus, by (app), $A . \sigma \vdash\left(M_{1} M_{2}\right): \tau$.

3) $M=\lambda . N$ : Let $A \vdash \lambda . N: \tau$. By (lambda), $\rho . A \vdash N: \mu$, where $\tau=\rho \rightarrow \mu$. By IH, $\rho . A . \sigma \vdash N: \mu$. Thus, by (lambda), A. $\sigma \vdash \lambda . N: \tau$.

4) $M=N[S]$ : Let $A \vdash N[S]: \tau$. By (clos), $A \vdash S \triangleright A^{\prime}$ and $A^{\prime} \vdash N: \tau$, for some $A^{\prime}$. Since $\|N[S]\|=\|N\|+\|S\|=0$, by Lemma $4, A . \sigma \vdash S \triangleright A^{\prime} . \sigma$. By IH, $A^{\prime} . \sigma \vdash N: \tau$. Thus, by (clos), $A . \sigma \vdash N[S]: \tau$.

Proof (Lemma 3 (Weakening for $T A_{\lambda \sigma}$ )). By induction on the structure of $M$ with subinduction on $\|\cdot\|$, having Lemmas 4 and 5 as induction base (IB).

1) $M=\underline{1}:$ Let $A \vdash \underline{1}: \tau$. By (var), $A=\tau$. $A^{\prime}$ for some $A^{\prime}$. Thus $A . \sigma \vdash \underline{1}: \tau$.

2) $M=\left(M_{1} M_{2}\right)$ : Let $A \vdash\left(M_{1} M_{2}\right): \tau$. By (app) one has that $A \vdash M_{1}: \rho \rightarrow \tau$ and $A \vdash M_{2}: \rho$, for some $\rho$. By IH on structure one has A. $\sigma \vdash M_{1}: \rho \rightarrow \tau$ and $A . \sigma \vdash M_{2}: \rho$. Thus, by (app), A. $\sigma \vdash\left(M_{1} M_{2}\right): \tau$.

3) $M=\lambda . N$ : Let $A \vdash \lambda . N: \tau$. By (lambda), $\rho . A \vdash N: \mu$, where $\tau=\rho \rightarrow \mu$. By IH, $\rho . A . \sigma \vdash N: \mu$. Thus, by (lambda), A. $\sigma \vdash \lambda . N: \tau$.

4) $M=N[S]$ : Let $A \vdash N[S]: \tau$. By (clos), $A \vdash S \triangleright A^{\prime}$ and $A^{\prime} \vdash N: \tau$, for some $A^{\prime}$. By IH, $A^{\prime} . \sigma \vdash N: \tau$. Substitution $S$ has to be examined. If $\|N\|>0$, then by $\mathrm{IH}$ on $\|\cdot\|$, as $\|N[S]\|>\|S\|$, one has that $A . \sigma \vdash S \triangleright A^{\prime} . \sigma$. Else, if $\|N\|=0$ then: 
- If $\|S\|=0$, then Lemma 4 can be applied.

- Otherwise, $S=P . T$ or $S=S_{1} \circ S_{2}$. If $S=P . T$, then by (cons), $A \vdash P: \rho$ and $A \vdash T \triangleright A^{\prime \prime}$, where $A^{\prime}=\rho . A^{\prime \prime}$. As $\|P\|,\|T\|<\|S\|=\|N[S]\|$, by IH on $\|\cdot\|, A . \sigma \vdash P: \rho$ and $A . \sigma \vdash T \triangleright A^{\prime \prime} . \sigma$. Thus, by (cons), $A . \sigma \vdash P . T \triangleright A^{\prime} . \sigma$. If $S=S_{1} \circ S_{2}$, then by (comp), $A \vdash S_{2} \triangleright A^{\prime \prime}$ and $A^{\prime \prime} \vdash S_{1} \triangleright A^{\prime}$, for some $A^{\prime \prime}$. If $\left\|S_{1}\right\|,\left\|S_{2}\right\|>0$, the result holds by IH on $\|\cdot\|$. Otherwise, at least one of the substitutions has $\|\cdot\|$ greater than 0 . Using induction on the structure of $S$ where $\|S\|>0$, the result holds. Then, $A . \sigma \vdash S_{2} \triangleright A^{\prime \prime} . \sigma$ and $A^{\prime \prime} . \sigma \vdash S_{1} \triangleright A^{\prime} . \sigma$. Thus, by (comp), $A . \sigma \vdash S_{1} \circ S_{2} \triangleright A^{\prime} . \sigma$.

Finally, by (clos), one has that $A . \sigma \vdash N[S]: \tau$.

\section{A.2 Proof of Correspondence}

Proof (Theorems 1, 3 and 5). The proofs are an adapted version of that given by Wells in [We2002]. Our adaptation deals with de Bruijn indices rather than variables and the proof for $\lambda \sigma$ has an adaptation to deal with substitutions too. Let $u \in\left\{\lambda d B, \lambda s_{e}, \lambda \sigma\right\}$ and $\mathcal{O}_{u}$ be the index updating operator of each calculus. In other words, $\mathcal{O}_{\lambda d B}(M)=M^{+}, \mathcal{O}_{\lambda s_{e}}(M)=\varphi_{0}^{2} M$ and $\mathcal{O}_{\lambda \sigma}(M)=M[\uparrow]$. Let $\mathcal{O}_{u}^{1}=\mathcal{O}_{u}$ and $\mathcal{O}_{u}^{n+1}(M)=\mathcal{O}_{u}\left(\mathcal{O}_{u}^{n}(M)\right)$. For a type $\tau$, let $\mathcal{T}(\tau)$ be the set of type variables occurring in $\tau$. For brevity, $\left.\underline{1}^{[} \uparrow^{n}\right]$ is denoted as $\underline{n+1}$.

$\Rightarrow$ proof: Let $\Theta_{u}=\left\langle A_{u}, \tau_{u}\right\rangle$ be a PT of some term $M_{u}$, according to Definitions 11, 13 and 15 , and $\Theta_{u}^{\prime}=\left\langle A_{u}^{\prime}, \tau_{u}^{\prime}\right\rangle$ be a typing of $M_{u}$. By the PT definition for each type system, there is a type substitution $s$ such that $s\left(A_{u}\right)=\left(A_{u}^{\prime}\right)_{\leq\left|A_{u}\right|}$. nil and $s\left(\tau_{u}\right)=\tau_{u}^{\prime}$. Since $T A_{u} \Vdash M: \Theta_{u}$ implies $T A_{u} \Vdash M: s\left(\Theta_{u}\right)$, for any type substitution $s$, we have $\Theta_{u} \leq_{T A_{u}} s\left(\Theta_{u}\right)$. By the weakening admissible rule for each type system $\left((\lambda d B\right.$-weak $),\left(\lambda s_{e}\right.$-weak $\left.)\right)$ and $(\lambda \sigma$-tweak $)$, we have $s\left(\Theta_{u}\right) \leq_{T A_{u}} \Theta_{u}^{\prime}$. Thus, $\Theta_{u}$ is PT of $M_{u}$, according to Definition 9.

The proof for a $\lambda \sigma$-substitution $S$ with PT $\Theta=\langle A, B\rangle$ according to Definiton 15 and typing $\Theta^{\prime}=\left\langle A^{\prime}, B^{\prime}\right\rangle$ is similar to the proof for $\lambda \sigma$-terms, using the proper weakening rule $(\lambda \sigma$-sweak).

$\Leftarrow$ proof: Let $\Theta_{u}=\left\langle A_{u}, \tau_{u}\right\rangle$ be a PT of some term $M_{u}$, according to Definitions 11, 13 and 15, and $\Theta_{u}^{\prime}=\left\langle A_{u}^{\prime}, \tau_{u}^{\prime}\right\rangle$ be a typing of $M_{u}$ which is not PT according to these definitions. Then, there exists a type substitution $s$ such that $s\left(A_{u}\right)=$ $\left(A_{u}^{\prime}\right)_{\leq\left|A_{u}\right|}$.nil and $s\left(\tau_{u}\right)=\tau_{u}^{\prime}$ and there does not exist any substitution $s^{\prime}$ such that $s^{\prime}\left(A_{u}^{\prime}\right)=\left(A_{u}\right)_{\leq\left|A_{u}^{\prime}\right|}$. nil and $s^{\prime}\left(\tau_{u}^{\prime}\right)=\tau_{u}$.

1. If $s\left(A_{u}\right) \neq A_{u}^{\prime}$, then $m_{u}=\left|A_{u}\right|<\left|A_{u}^{\prime}\right|$. Let $N_{u}=\left(\lambda \cdot \mathcal{O}_{u}\left(M_{u}\right) m_{u}+1\right)$.

2. If $s\left(A_{u}\right)=A_{u}^{\prime}$, let $\alpha$ be a type variable. Define the functions $\overline{\phi_{1}^{u}, \phi_{2}^{u}}$ by:

$$
\begin{aligned}
& \phi_{1}^{u}(\alpha, \alpha)=\lambda \cdot \lambda \cdot(\underline{1}(\underline{2} \underline{4})(\underline{2} \underline{3})) \\
& \phi_{1}^{u}(\sigma \rightarrow \tau, \alpha)= \begin{cases}\lambda . \lambda .\left(\underline{1}(\underline{3} \underline{2})\left(\mathcal{O}_{u}^{3}\left(\lambda \cdot \phi_{1}^{u}(\sigma, \alpha)\right) \underline{2}\right)\right), & \text { if } \alpha \in \mathcal{T}(\sigma) \\
\lambda .\left(\mathcal{O}_{u}^{2}\left(\lambda . \phi_{1}^{u}(\tau, \alpha)\right)(\underline{2} \underline{1})\right), & \text { otherwise }\end{cases}
\end{aligned}
$$




$$
\begin{aligned}
\phi_{2}^{u}(\alpha, \alpha) & =\lambda \cdot \lambda \cdot(\underline{1}(\underline{2} \underline{3})(\underline{2} \underline{4})) \\
\phi_{2}^{u}(\sigma \rightarrow \tau, \alpha) & = \begin{cases}\lambda . \lambda .\left(\underline{1}(\underline{4} \underline{2})\left(\mathcal{O}_{u}^{2}\left(\lambda . \phi_{1}^{u}(\sigma, \alpha)\right) \underline{2}\right)\right), & \text { if } \alpha \in \mathcal{T}(\sigma) \\
\lambda .\left(\mathcal{O}_{u}\left(\lambda \cdot \phi_{1}^{u}(\tau, \alpha)\right)(\underline{3} \underline{1})\right), & \text { otherwise }\end{cases}
\end{aligned}
$$

(a) Suppose $s\left(\alpha_{u}\right)$ is not a type variable for $\alpha_{u} \in \mathcal{T}\left(\Theta_{u}\right)$

i. Suppose $\alpha_{u} \in \mathcal{T}\left(\tau_{u}\right)$.

Let $N_{u}=\left(\lambda .\left(\lambda . \underline{2} \lambda .\left(\mathcal{O}_{u}\left(\lambda . \phi_{2}^{u}\left(\tau_{u}, \alpha_{u}\right)\right) \lambda . \underline{2}\right)\right) M_{u}\right)$.

ii. Suppose $\alpha_{u} \in \mathcal{T}\left(\left(A_{u}\right)_{i_{u}}\right)$.

Let $N_{u}=\left(\lambda . \mathcal{O}_{u}\left(M_{u}\right) \lambda .\left(\lambda . \lambda . \phi_{2}^{u}\left(\left(A_{u}\right)_{i_{u}}, \alpha_{u}\right) i_{u}+1 \lambda . \underline{2}\right)\right)$.

(b) Suppose $s\left(\alpha_{u}^{1}\right)=s\left(\alpha_{u}^{2}\right)=\beta$ for distinct $\alpha_{u}^{1}, \alpha_{u}^{2} \in \overline{\mathcal{T}}\left(\Theta_{u}\right)$

i. Suppose $\alpha_{u}^{j} \in \mathcal{T}\left(\left(A_{u}\right)_{i_{u, j}}\right)$ for $j \in\{1,2\}$.

Let $P_{u}^{j}=\left(\lambda . \phi_{1}^{u}\left(\left(A_{u}\right)_{i_{u, j}}, \alpha_{u}^{j}\right) i_{u, j}+1\right)$

and $P_{u}=\lambda . \lambda .\left(\underline{1} \mathcal{O}_{u}\left(P_{u}^{1}\right) \mathcal{O}_{u}\left(\overline{\left.\left.P_{u}^{2}\right)\right)}\right.\right.$. Let $N_{u}=\left(\lambda . \lambda . \underline{2} M_{u} P_{u}\right)$.

ii. Suppose $\alpha_{u}^{1} \in \mathcal{T}\left(\left(A_{u}\right)_{i_{u}}\right)$ and $\alpha_{u}^{2} \in \mathcal{T}\left(\tau_{u}\right)$.

Let $P_{u}=\lambda . \lambda .\left(\underline{1}\left(\mathcal{O}_{u}\left(\lambda \cdot \phi_{1}^{u}\left(\left(A_{u}\right)_{i_{u}}, \alpha_{u}^{1}\right)\right) \underline{i_{u}+3}\right) \mathcal{O}_{u}\left(\phi_{2}^{u}\left(\tau_{u}, \alpha_{u}^{2}\right)\right)\right)$ and $N_{u}=\left(\lambda .\left(\lambda . \underline{2} P_{u}\right) M_{u}\right)$.

iii. Suppose $\alpha_{u}^{i} \in \mathcal{T}\left(\tau_{u}\right)$ for $i \in\{1,2\}$.

Let $P_{u}=\lambda . \lambda .\left(\underline{1} \mathcal{O}_{u}\left(\phi_{2}^{u}\left(\tau_{u}, \alpha_{u}^{1}\right)\right) \mathcal{O}_{u}\left(\phi_{2}^{u}\left(\tau_{u}, \alpha_{u}^{2}\right)\right)\right)$

and $N_{u}=\left(\lambda .\left(\lambda . \underline{2} P_{u}\right) M_{u}\right)$.

Then, $N_{u} \in \operatorname{Terms}_{T A_{u}}\left(\Theta_{u}^{\prime}\right) \backslash \operatorname{Terms}_{T A_{u}}\left(\Theta_{u}\right)$. Thus, $\Theta_{u}^{\prime} \not_{T A_{u}} \Theta_{u}$.

As consequence, if $\Theta_{u}^{\prime}$ is not PT according to Definitions 11, 13 and $15, \Theta_{u}^{\prime}$ is not PT according to Definition 9.

Let $M$ be a $\lambda \sigma$-substitution $S$ and $\Theta=\langle A, B\rangle$ be a PT of $S$, according to Definition 15, and $\Theta^{\prime}=\left\langle A^{\prime}, B^{\prime}\right\rangle$ be a typing of $S$ which is not PT according to this definition. Then, there is a type substitution $s$ such that $s(A)=A_{\leq|A|}^{\prime}$.nil and $s(B)=B_{\leq|B|}^{\prime}$. nil and there is no substitution $s^{\prime}$ such that $s^{\prime}\left(A^{\prime}\right)=A_{\leq\left|A^{\prime}\right|}$. nil and $s^{\prime}\left(B^{\prime}\right)=B_{\leq\left|B^{\prime}\right|}$.nil.

1. Suppose $s(A) \neq A^{\prime}$. Then, $m=|A|<\left|A^{\prime}\right|$.

Let $S_{i}=\left(\underline{1} \cdot \underline{2} \cdot \cdots \cdot \underline{m+1} \cdot \uparrow^{m+1}\right)$ and $T=S \circ S_{i}$.

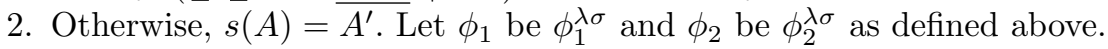

(a) Suppose $s(\alpha)$ is not a type variable for $\alpha \in \mathcal{T}(\Theta)$

i. Suppose $\alpha \in \mathcal{T}\left(B_{i}\right)$. Let $N=\left(\lambda .\left(\lambda . \underline{2} \lambda .\left(\left(\lambda . \phi_{2}\left(B_{i}, \alpha\right)\right)[\uparrow] \lambda . \underline{2}\right)\right) \underline{i}\right)$ and let $S_{i}^{\prime}=\left(\underline{1} \cdot \underline{2} \cdot \cdots \cdot \underline{i-1} \cdot N \cdot \uparrow^{i}\right)$. Let $T=S_{i}^{\prime} \circ S$.

ii. Suppose $\alpha \in \mathcal{T}\left(A_{i}\right)$. Let $N$ and $S_{i}^{\prime}$ be as above. Let $T=S \circ S_{i}^{\prime}$.

(b) Suppose $s\left(\alpha_{1}\right)=s\left(\alpha_{2}\right)=\beta$ for distinct $\alpha_{1}, \alpha_{2} \in \mathcal{T}(\Theta)$

i. Suppose $\alpha_{j} \in \mathcal{T}\left(A_{i_{j}}\right)$ for $j \in\{1,2\}$. Let $P_{j}=\left(\lambda . \phi_{1}\left(A_{i_{j}}, \alpha_{j}\right) i_{j}+1\right)$ and $P=\lambda . \lambda .\left(\underline{1} P_{1}[\uparrow] P_{2}[\uparrow]\right)$. Let $N_{j}=\left(\lambda . \lambda . \underline{2} i_{j} P\right)$, where $j \in \overline{\{1,2\}}$ and let $S_{i_{j}}=\left(\underline{1} \cdot \underline{2} \cdot \cdots \cdot i_{j}-1 \cdot N_{j} \cdot \uparrow^{i_{j}}\right)$. Let $T=\underline{T}=S \circ S_{i_{j}}$.

ii. Suppose $\alpha_{j} \in \mathcal{T}\left(\overline{\left.B_{i_{j}}\right),} j \in\{1,2\}\right.$. Let $P_{j}=\left(\lambda . \phi_{1}\left(B_{i_{j}}, \alpha_{j}\right) \underline{i_{j}+1}\right)$. Then, for $P, N_{j}$ and $S_{i_{j}}$ as above, let $T=S_{i_{j}} \circ S$.

iii. Suppose $\alpha_{1} \in \mathcal{T}\left(A_{i}\right)$ and $\alpha_{2} \in \mathcal{T}\left(B_{j}\right)$. Let $N=(\lambda .(\lambda . \underline{2} P) \underline{j}[S])$, where $P=\lambda . \lambda .\left(\underline{1}\left(\left(\lambda . \phi_{1}\left(A_{i}, \alpha_{1}\right)\right)[\uparrow] \underline{i+3}\right) \phi_{2}\left(B_{j}, \alpha_{2}\right)[\uparrow]\right)$. Let $T=$ $\left(\underline{1}[S] . \underline{2}[S] . \cdots, \underline{j-1}[S] . N \cdot\left(\uparrow^{j} \circ S\right)\right)$. 
Then, $T \in \operatorname{Terms}_{T A_{\lambda \sigma}}\left(\Theta^{\prime}\right) \backslash \operatorname{Terms}_{T A_{\lambda \sigma}}(\Theta)$. Thus, $\Theta^{\prime} \not_{T A_{\lambda \sigma}} \Theta$

As consequence, if a typing $\Theta^{\prime}$ of some $\lambda \sigma$-substitution is not PT according to Definition 15, then $\Theta^{\prime}$ is not PT according to Definition 9.

\section{A.3 Proof of PT}

Proof (Theorems 2, 4 and 6). Let $M$ be any term (expression in $\lambda \sigma$ ) and $M^{\prime}$ its decorated version. Let $R_{0}$ be the set of all sub-terms (sub-expressions) of $M^{\prime}$. Starting with the pair $\left\langle\left\langle R_{0}, \emptyset\right\rangle\right\rangle$ and applying the rules of the type inference algorithm in Table 3, 4 or 5 one obtains a final pair after a finite number of steps, because after each step the number of elements in the set of decorated sub-terms(sub-expressions) of the pair is decremented. By the uniqueness in the decomposition of the sub-terms (sub-expressions) in each calculus, a unique rule can be applied to each element of $R_{0}$. Thus, the process finishes with a pair $\left\langle\left\langle\emptyset, E_{f}\right\rangle\right\rangle$, where $E_{f}$ is a set of first-order equations over context and type variables, according to the rules of the type systems $T A_{\lambda d B}, T A_{\lambda s_{e}}$ and $T A_{\lambda \sigma}$ respectively. An adequate first-order unification algorithm, e.g. see [Hi97], is then applied. And by the correctness, completeness and uniqueness of first-order unification, one has that the algorithm will find an mgu if $M$ is typable. Otherwise, the algorithm will report that there are no unifiers. Consequently, the typing systems $T A_{\lambda d B}, T A_{\lambda s_{e}}$ and $T A_{\lambda \sigma}$ satisfy PT. 